\title{
On the Uniqueness of Diffeomorphism Symmetry in Conformal Field Theory
}

\author{
SEBASTIANO CARPI * \\ Dipartimento di Scienze \\ Università "G. d'Annunzio" di Chieti-Pescara \\ Viale Pindaro 87, I-65127 Pescara, Italy \\ E-mail: carpi@sci.unich.it \\ MIHÁLY WEINER \\ Dipartimento di Matematica \\ Università di Roma "Tor Vergata" \\ Via della Ricerca Scientifica 1, I-00133 Roma, Italy \\ E-mail: weiner@mat.uniroma2.it
}

\begin{abstract}
A Möbius covariant net of von Neumann algebras on $S^{1}$ is diffeomorphism covariant if its Möbius symmetry extends to diffeomorphism symmetry. We prove that in case the net is either a Virasoro net or any at least 4-regular net such an extension is unique: the local algebras together with the Möbius symmetry (equivalently: the local algebras together with the vacuum vector) completely determine it. We draw the two following conclusions for such theories. (1) The value of the central charge $c$ is an invariant and hence the Virasoro nets for different values of $c$ are not isomorphic as Möbius covariant nets. (2) A vacuum preserving internal symmetry always commutes with the diffeomorphism symmetries. We further use our result to give a large class of new examples of nets (even strongly additive ones), which are not diffeomorphism covariant; i.e. which do not admit an extension of the symmetry to $\operatorname{Diff}^{+}\left(S^{1}\right)$.
\end{abstract}

\footnotetext{
*Supported in part by the Italian MIUR and GNAMPA-INDAM.
} 


\section{Introduction}

This paper is motivated by the following question: is the $\operatorname{Diff}^{+}\left(S^{1}\right)$ symmetry, or the corresponding Virasoro algebra symmetry, exhibited by 2-dimensional Quantum Field Theory models unique?

We shall give a precise formulation to this question in the framework of Algebraic Quantum Field Theory (see the book of R. Haag [13]). In this framework a chiral 2-dimensional quantum field theory is commonly described by means of a Möbius covariant net of von Neumann algebras on $S^{1}$. The net is said to be diffeomorphism covariant if the corresponding positive energy representation of the Möbius group has an extension to a (strongly continuous) projective unitary representation of $\operatorname{Diff}^{+}\left(S^{1}\right)$ that acts covariantly on the von Neumann algebras associated to the intervals of $S^{1}$ and that is compatible with the local structure of the net (see Sect. 2 for the precise definition). It is known that this extension does not exist in general (see e.g. [12, 19] and cf. also Sect. 6 below) but to the best of our knowledge no results about its uniqueness appears in the literature despite the fact that this problem seems to be very natural. Besides of its mathematical naturalness the relevance of the above uniqueness is strengthened by the increasing importance played in the past years by diffeomorphism symmetry of nets of von Neumann algebras on $S^{1}$ in the investigation of the structural properties of two-dimensional conformal field theories, see e.g. [16, 17, 20, 31, 4, 22.

The main result of this paper is the proof that for large class of diffeomorphism covariant nets on $S^{1}$ the $\operatorname{Diff}^{+}\left(S^{1}\right)$ symmetry is unique in the sense explained above and hence that it is completely determined by the underlying structure of the Möbius covariant net. More precisely we prove uniqueness for all Virasoro nets, namely the nets generated by the zero-energy representations of $\operatorname{Diff}^{+}\left(S^{1}\right)$, (Theorem 3.3) and for all 4-regular diffeomorphism covariant nets on $S^{1}$ (Theorem 5.5). The latter class (see Sect. 2 for the definition) includes every strongly additive diffeomorphism covariant net on $S^{1}$ and hence every diffeomorphism covariant net which is completely rational in the sense of [18], the nets generated by chiral current algebras [1, 12, 27, 30] and their orbifold subnets 31. Since the Möbius symmetry of a given net on $S^{1}$ is completely determined by the vacuum vector [9, Theorem 2.19] our result shows that in the above cases the Diff ${ }^{+}\left(S^{1}\right)$ symmetry of the net is also determined by this vector. Note also that the known examples of Möbius covariant nets which are not 4-regular are not diffeomorphism covariant (see [12, 19]), so our uniqueness result could apply to every diffeomorphism covariant net on $S^{1}$.

Let us now discuss some consequences of our results. Firstly the uniqueness in the case of Virasoro nets implies that two Virasoro nets cannot be isomorphic as Möbius covariant nets on the circle if they have different central charges (Corollary [3.4), a fact that seems to be widely expected (see e.g. the introduction of [1]) but that has not been explicitly stated in the literature. Similarly two 4-regular diffeomorphism covariant nets cannot be isomorphic as Möbius covariant nets on $S^{1}$ if the corresponding representations of $\operatorname{Diff}^{+}\left(S^{1}\right)$ are not unitarily equivalent and in particular if they have a different central charge (Corollary [5.6). Another interesting consequence is that we 
have a model independent proof of the fact that diffeomorphisms symmetries commute with vacuum preserving internal symmetries of a given 4-regular net (Corollary [5.8). Finally we apply our result to show that the tensor product of an infinite sequence of 4-regular diffeomorphism covariant net on $S^{1}$ is not diffeomorphism covariant (Theorem 6.1).

This paper is organized as follows. In Sect. 2 we discuss various preliminaries about Möbius covariant nets on $S^{1}$, subnets and diffeomorphism covariance together with its relation to the Virasoro algebra. Almost all this facts are already carefully discussed in the literature and we include them only to fix the notation and to keep the paper reasonably self-contained. In Sect. 3 we prove the uniqueness of the $\operatorname{Diff}^{+}\left(S^{1}\right)$ symmetry in the case of Virasoro nets. The result is obtained by showing in a rather direct way that the (chiral) stress-energy tensors associated to two representations of $\operatorname{Diff}^{+}\left(S^{1}\right)$ making a Virasoro net diffeomorphism covariant have to coincide. In Sect. 4 we show that the maps corresponding to projective unitary representations of $\operatorname{Diff}^{+}\left(S^{1}\right)$ continuously extend to a certain family of nonsmooth diffeomorphisms in an appropriate topology. The result is proved at the Lie algebra level. Since the estimates in the paper of Goodmann and Wallach [10] are not sufficient for our purpose we need a more detailed analysis. In particular we cannot use directly Nelson's commutator theorem [24, 26] to show that the operators involved are self-adjoint but we find an estimate involving the contraction semigroup associated to the conformal Hamiltonian $L_{0}$ which allows us to demonstrate self-adjointness following the ideas of the paper of E. Nelson [24]. In Sect. 5 we use the results of Sect. 4 to construct a nontrivial local operator which belongs to the Virasoro subnet associated to an arbitrary representation of $\operatorname{Diff}^{+}\left(S^{1}\right)$ making a given 4-regular net diffeomorphism covariant. This construction, together with the main result in Sect. 3] and the minimality property of Virasoro nets proved in [2] allows us to reach the main objective of this paper, namely the uniqueness of the $\operatorname{Diff}^{+}\left(S^{1}\right)$ symmetry for 4-regular nets. Finally in Sect. 6] we discuss the above mentioned application of our main result to the case of infinite tensor products of nets.

\section{Preliminaries}

\subsection{Möbius covariant nets}

Let $\mathcal{J}$ be the set of open, nonempty and nondense arcs (also called: open proper arcs or open proper intervals) of the unit circle $S^{1}=\{z \in \mathbb{C}:|z|=1\}$. A Möbius covariant net on $S^{1}$ is a map $\mathcal{A}$ which assigns to every open proper arc $I \subset S^{1}$ a von Neumann algebra $\mathcal{A}(I)$ acting on a fixed complex, infinite dimensional separable Hilbert space $\mathcal{H}_{\mathcal{A}}$ ("the vacuum Hilbert space of the theory"), together with a given strongly continuous representation $U$ of $\mathrm{Möb} \simeq \operatorname{PSL}(2, \mathbb{R})$, the group of Möbius transformations ${ }^{1}$ of the unit circle $S^{1}$ satisfying for all $I_{1}, I_{2}, I \in \mathcal{J}$ and $\varphi \in$ Möb the following properties:

\footnotetext{
${ }^{1}$ diffeomorphisms of $S^{1}$ of the form $z \mapsto \frac{a z+b}{\bar{b} z+\bar{a}}$ with $a, b \in \mathbb{C},|a|^{2}-|b|^{2}=1$.
} 
(i) Isotony.

$$
I_{1} \subset I_{2} \Rightarrow \mathcal{A}\left(I_{1}\right) \subset \mathcal{A}\left(I_{2}\right),
$$

(ii) Locality.

$$
I_{1} \cap I_{2}=\emptyset \Rightarrow\left[\mathcal{A}\left(I_{1}\right), \mathcal{A}\left(I_{2}\right)\right]=0,
$$

(iii) Covariance.

$$
U(\varphi) \mathcal{A}(I) U(\varphi)^{-1}=\mathcal{A}(\varphi(I)),
$$

(iv) Positivity of the energy. The representation $U$ is of positive energy type: the conformal Hamiltonian $L_{0}$, defined by $U\left(\theta_{\alpha}\right)=e^{i \alpha L_{0}}$ where $\theta_{\alpha} \in$ Möb is the anticlockwise rotation by degree $\alpha$, is positive.

(v) Existence and uniqueness of the vacuum. There exists a unique (up to phase) unit vector $\Omega \in \mathcal{H}_{\mathcal{A}}$ called the "vacuum vector" which is invariant under the action of $U$. (Equivalently: up to phase there exists a unique unit vector $\Omega$ that is of zero-energy for $U$; i.e. eigenvector of $L_{0}$ with eigenvalue zero.)

(vi) Cyclicity of the vacuum. $\Omega$ is cyclic for the algebra $\mathcal{A}\left(S^{1}\right)=\bigvee_{I \in \mathcal{J}} \mathcal{A}(I)$.

Some consequences of the axioms are [9, 11, 8]:

(i) Reeh-Schlieder property. $\Omega$ is a cyclic and separating vector of the algebra $\mathcal{A}(I)$ for every $I \in \mathcal{J}$.

(ii) Bisognano-Wichmann property.

$$
U\left(\Lambda_{I}(2 \pi t)\right)=\Delta_{I}^{i t}
$$

where $\Delta_{I}$ is the modular operator associated to $\mathcal{B}(I)$ and $\Omega$, and $\Lambda_{I}$ is the oneparameter group of Möbius transformations preserving the interval $I$ (the dilations associated to $I$ ) with the "right" parametrization (see e.g. [12]).

(iii) Haag duality. For every $I \in \mathcal{J}$

$$
\mathcal{A}(I)^{\prime}=\mathcal{A}\left(I^{\prime}\right)
$$

where $I^{\prime}$ denotes the interior of the complement set of $I$ in $S^{1}$.

(iv) Irreducibility. $\mathcal{A}\left(S^{1}\right)=\bigvee_{I \in \mathcal{J}} \mathcal{A}(I)=\mathrm{B}\left(\mathcal{H}_{\mathcal{A}}\right)$, where $\mathrm{B}\left(\mathcal{H}_{\mathcal{A}}\right)$ denotes the algebra of all bounded linear operators on $\mathcal{H}_{\mathcal{A}}$.

(v) Additivity. If $\mathcal{S} \subset \mathcal{J}$ is a covering of the interval $I$ then

$$
\mathcal{A}(I) \subset \bigvee_{J \in \mathcal{S}} \mathcal{A}(J)
$$


As a consequence of the Bisognano-Wichmann property, since Möb is generated by the dilations (associated to different intervals), the representation $U$ is completely determined by the local algebras and the vacuum vector via modular structure. Thus, we may say that there is a kind of uniqueness regarding the representation of the Möbius group.

According to the last property (additivity) and the isotony, if $I_{1}, I_{2}, I \in \mathcal{J}$ are such that $I_{1} \cup I_{2}=I$ then $\mathcal{A}\left(I_{1}\right) \vee \mathcal{A}\left(I_{2}\right)=\mathcal{B}(I)$. In many (but not all) physically interesting model an even stronger additivity property holds. The net $\mathcal{A}$ is said to be strongly additive, if $\mathcal{A}\left(I_{1}\right) \vee \mathcal{A}\left(I_{2}\right)=\mathcal{A}(I)$ whenever $I_{1}, I_{2}$ are the connected components of $I \backslash\{p\}$ where $p$ is a point of the open interval $I$.

For an $n=2,3, .$. the net $\mathcal{A}$ is said to be $\mathbf{n}$-regular, if whenever we remove $n$ points from the circle the algebras associated to the remaining intervals generate the whole of $\mathcal{A}\left(S^{1}\right)=\mathrm{B}\left(\mathcal{H}_{\mathcal{A}}\right)$. By isotony $n$-regularity is a stronger property then $m$-regularity if $n>m$, and by Haag duality every Möbius covariant net is at least 2-regular. Strong additivity is of course stronger than $n$-regularity for any $n$.

All these properties are indeed "additional": there are Möbius covariant nets which are not even 3-regular (see the examples in [12]).

\subsection{Diffeomorphism covariance and the Virasoro nets}

Let Diff ${ }^{+}\left(S^{1}\right)$ be the group of orientation preserving (smooth) diffeomorphisms of the circle. It is an infinite dimensional Lie group with Lie algebra identified with the real topological vector space $\operatorname{Vect}\left(S^{1}\right)$ of smooth real vectors fields on $S^{1}$ with the usual $C^{\infty}$ topology [23, Sect. 6] endowed with the bracket given by the negative of the usual brackets of vector fields. In this paper often we shall think of a the vector field symbolically written as $f\left(e^{i \vartheta}\right) \frac{d}{d \vartheta} \in \operatorname{Vect}\left(S^{1}\right)$ as the corresponding real function $f$. Also we shall use the notation $f^{\prime}$ (calling it simply the derivative) for the function on the circle obtained by derivating with respect to the angle: $f^{\prime}\left(e^{i \theta}\right)=\left.\frac{d}{d \alpha} f\left(e^{i \alpha}\right)\right|_{\alpha=\theta}$.

A strongly continuous projective unitary representation $V$ of $\operatorname{Diff}^{+}\left(S^{1}\right)$ on a Hilbert space $\mathcal{H}$ is a strongly continuous $\operatorname{Diff}^{+}\left(S^{1}\right) \rightarrow \mathcal{U}(\mathcal{H}) / \mathbb{T}$ homomorphism. The restriction of $V$ to Möb $\subset \operatorname{Diff}^{+}\left(S^{1}\right)$ always lifts to a unique strongly continuous unitary representation of the universal covering group $\widetilde{\mathrm{Möb}}$ of Möb. $V$ is said to be of positive energy type, if its conformal Hamiltonian $L_{0}$, defined by the above representation of Möb (similarly as in case of a representation of the group Möb) has nonnegative spectrum. In this case we shall simply say that $V$ is a positive energy representation of $\operatorname{Diff}^{+}\left(S^{1}\right)$.

Sometimes for a $\gamma \in \operatorname{Diff}^{+}\left(S^{1}\right)$ we shall think of $V(\gamma)$ as a unitary operator. Although there are more than one ways to fix the phases, note that expressions like $\operatorname{Ad}(V(\gamma))$ or $V(\gamma) \in \mathcal{M}$ for a von Neumann algebra $\mathcal{M} \subset \mathrm{B}(\mathcal{H})$ are unambiguous. We shall also say that $V$ is an extension of the unitary representation $U$ of Möb if we can arrange the phases in such a way that $V(\varphi)=U(\varphi)$, or without mentioning phases: $\operatorname{Ad}(V(\varphi))=\operatorname{Ad}(U(\varphi))$, for all $\varphi \in \operatorname{Möb}$.

We have to keep in mind that after choosing phases the equality of $V$ to another

projective representation $\tilde{V}$ means that $V(\gamma)^{*} \tilde{V}(\gamma)$ is a multiple of the identity (and 
not necessary the identity) for all $\gamma \in \operatorname{Diff}^{+}\left(S^{1}\right)$.

Definition 2.1. A Möbius covariant net $(\mathcal{A}, U)$ is diffeomorphism covariant if there is a strongly continuous projective unitary representation $V$ of $\operatorname{Diff}^{+}\left(S^{1}\right)$ on $\mathcal{H}_{\mathcal{A}}$ such that for all $\gamma \in \operatorname{Diff}^{+}\left(S^{1}\right)$ and $I, J \in \mathcal{J}$

$$
\begin{aligned}
& \text { 1. } \gamma \in M \ddot{o} b \Rightarrow \operatorname{Ad}(V(\gamma))=\operatorname{Ad}(U(\gamma)) \\
& \text { 2. }\left.\gamma\right|_{I}=\left.\mathrm{id}_{I} \Rightarrow \operatorname{Ad}(\mathrm{V}(\gamma))\right|_{\mathcal{A}(\mathrm{I})}=\mathrm{id}_{\mathcal{A}(\mathrm{I})} . \\
& \text { 3. } \gamma(I)=J \Rightarrow V(\gamma) \mathcal{A}(I) V(\gamma)^{*}=\mathcal{A}(J) .
\end{aligned}
$$

In particular $V$ is a positive energy representation of $\operatorname{Diff}^{+}\left(S^{1}\right)$ extending $U$.

Note that as a consequence of Haag duality and of the second of the above listed properties, if a diffeomorphism localized in the interval $I$ - i.e. it acts trivially (identically) elsewhere - then the corresponding unitary is also localized in $I$ in the sense that it belongs to $\mathcal{A}(I)$.

The majority of the known examples of "interesting" conformal field theories are diffeomorphism covariant. In fact it may turn out to be that under some "regularity" condition imposed on the net diffeomorphism covariance is automatic. In this respect the examples of non-diffeomorphism covariant nets which we shall give in the last section are useful in showing that for example strong additivity in itself is not a sufficient condition. (Up to the knowledge of the authors, there have been no previous examples of strongly additive nets that are not diffeomorphism covariant.)

We now briefly describe the irreducible positive energy representations of $\operatorname{Diff}^{+}\left(S^{1}\right)$ - for fixing notations rather than to introduce them - and the so-called Virasoro nets. (Find more in [14, 10], 28] and 6], for example.) For certain values of the central charge $c>0$ and the lowest weight $h \geq 0$ there is a positive energy projective representation denoted by $V_{(c, h)}$ on the Hilbert space $\mathcal{H}_{(c, h)}$. In $V_{(c, h)}$ the spectrum of the conformal Hamiltonian $\operatorname{Sp}\left(L_{0}\right)=\{h, h+1, h+2, .$.$\} unless h=0$ in which case the value $h+1=1$ is missing from it; all these corresponding of course to eigenvalues, only. The eigenspace associated to the value $h$ is one-dimensional. We shall denote by $\Phi$ the (up-to-phase) unique unit vector in this eigenspace. The dense subspace $\mathcal{D}_{\text {fin }}$ consisting of the linear combinations of the eigenvectors will be called the space of "finite-energy" vectors. The representation via infinitesimal generators defines an irreducible unitary lowest weight representation $\left\{L_{n}: n \in \mathbb{Z}\right\}$ of the Virasoro algebra satisfying for all natural numbers $n, m$

1. (core) $\mathcal{D}_{\text {fin }}$ is a core and invariant for the closed operator $L_{n}$

2. (lowest weight) if $n>0$ then $L_{n} \Phi=0$

3. (unitarity) $L_{n}^{*}=L_{-n}$

4. (Virasoro algebra relations) on the common invariant core $\mathcal{D}_{\text {fin }}$

$$
\left[L_{n}, L_{m}\right]=(n-m) L_{n+m}+\frac{c}{12}\left(n^{3}-n\right) \delta_{-m, n} \mathbb{1} .
$$


The correspondence between the infinitesimal generators and the representation is the following. For an $f \in \operatorname{Vect}\left(S^{1}\right) \equiv C^{\infty}\left(S^{1}, \mathbb{R}\right)$ real vector field with Fourier coefficients

$$
\hat{f}_{n}=\frac{1}{2 \pi} \int e^{-i n \alpha} f\left(e^{i \alpha}\right) d \alpha \quad(n \in \mathbb{Z})
$$

the operator $T_{0}(f)$ on domain $\mathcal{D}_{\text {fin }}$ given by

$$
T_{0}(f)=\sum_{n \in \mathbb{Z}} \hat{f}_{n} L_{n}
$$

is well-defined and essentially self-adjoint. Then, denoting $T(f)$ the self-adjoint operator obtained by the closure of $T_{0}(f)$ and omitting $c$ and $h$ indices, we have that

$$
e^{i T(f)}=V(\operatorname{Exp}(\mathrm{f}))
$$

after an appropriate choice of the phase of the right hand side. Via this relation (and the conditions listed above) $V$ and the operators $L_{n}(n \in \mathbb{Z})$ completely determine each other. $T$ is called the stress-energy tensor, it can be looked upon as an operator valued distribution.

Remark. Note that in the literature usually the operators $L_{n} n \in \mathbb{Z}$ are not taken as closed operators, i.e. our notations stand for the closure of those.

The possible values of $c$ are $\{1-6 /((m+2)(m+3)) \mid m=1,2,3, .$.$\} and c \geq 1$, and for all these $h=0$ is a possible lowest weight. In case of $h=0$, we shall denote the representation simply by $V_{c}$ (omiting the zero in the subscript), and by $\Omega$ the (upto-phase) unique unit zero-energy vector (omiting even the subscript " $c$ "). For every $I \in \mathcal{J}$ with the

Definition 2.2. $\mathcal{A}_{\mathrm{Vir}, \mathrm{c}}(I)=\left\{V_{c}(\gamma) \in \mathrm{B}\left(\mathcal{H}_{(c, 0)}\right)|\gamma|_{I^{\prime}}=\mathrm{id}_{\mathrm{I}^{\prime}}\right\}^{\prime \prime}$

the net $\mathcal{A}_{\mathrm{Vir}, \mathrm{c}}$ with the representation of Möb obtained by restriction of $V_{c}$ is a Möbius covariant net on $S^{1}$, which is also diffeomorphism covariant with respect to the representation $V_{c}$. This is the so-called Virasoro net.

With what was said before we have described all irreducible positive energy representations of the diffeomorphism group: recall (4, Theorem A.1]) that an irreducible positive energy representation of $\operatorname{Diff}^{+}\left(S^{1}\right)$ is equivalent to $V_{(c, h)}$ for some value of $c$ and $h$. The proof in [4] is based on results in [21].

\subsection{Subnets}

A (Möbius covariant) subnet of the Möbius covariant net $(\mathcal{A}, U)$ is an assignment of nontrivial von Neumann algebras to the open proper arcs of the circle $I \mapsto \mathcal{B}(I)$ such that for all $I_{1}, I_{2}, I \in \mathcal{J}$ and $\varphi \in$ Möb

(i) $\mathcal{B}(I) \subset \mathcal{A}(I)$ 
(ii) $I_{1} \subset I_{2} \Rightarrow \mathcal{B}\left(I_{1}\right) \subset \mathcal{B}\left(I_{2}\right)$

(iii) $\varphi \in \mathrm{Möb} \Rightarrow U(\varphi) \mathcal{B}(I) U(\varphi)^{*}=\mathcal{B}(\varphi(I))$.

We shall use the notation $\mathcal{B} \subset \mathcal{A}$ for subnets.

A subnet $\mathcal{B} \subset \mathcal{A}$ which is proper (namely it does not coincide with $\mathcal{A}$ ) is not a Möbius covariant net in the precise sense of the definition because we do not have the cyclicity of the vacuum with respect to $\mathcal{B}$. However, this inconvenience can be overcome by restriction to the Hilbert space

$$
\mathcal{H}_{\mathcal{B}}=\overline{\mathcal{B}\left(S^{1}\right) \Omega}=\overline{\bigvee_{I \in \mathcal{J}} \mathcal{B}(I) \Omega}
$$

where $\Omega$ is the vacuum vector. It is evident that $\mathcal{H}_{\mathcal{B}}$ is invariant for $U$. The map $\left.I \mapsto \mathcal{B}(I)\right|_{\mathcal{H}_{\mathcal{B}}}$ together with the restriction of $U$ onto $\mathcal{H}_{\mathcal{B}}$ is a Möbius covariant net. Rather direct consequences of the definition and of the properties of Möbius covariant nets (such as for example the Reeh-Schlieder and Haag property) are:

(i) for any $I \in \mathcal{J}$ the restriction map from $\mathcal{B}(I)$ to $\left.\mathcal{B}(I)\right|_{\mathcal{H}_{\mathfrak{B}}}$ is an isomorphism between von Neumann algebras,

(ii) the map $\left.A \mapsto P_{\mathcal{B}} A\right|_{\mathcal{H}_{\mathcal{B}}}$ where $P_{\mathcal{B}}$ is the orthogonal projection onto $\mathcal{H}_{\mathcal{B}}$ and $A \in$ $\mathcal{A}(I)$ for a fixed $I \in \mathcal{J}$ defines a faithful normal conditional expectation from $\mathcal{A}(I)$ to $\mathcal{B}(I)$ after identifying $\mathcal{B}(I)$ with $\left.\mathcal{B}(I)\right|_{\mathcal{H}_{\mathcal{B}}}$ using point (i),

(iii) $\mathcal{B}\left(S^{1}\right) \cap \mathcal{A}(I)=\mathcal{B}(I)$ for all $I \in \mathcal{J}$.

A fundamental example of a subnet which we shall briefly describe here is the one determined by the stress-energy tensor in a diffeomorphism covariant theory. Suppose the net $(\mathcal{A}, U)$ is diffeomorphism covariant with respect to the strongly continuous projective representation $V$ of $\operatorname{Diff}^{+}\left(S^{1}\right)$. Then the formula

$$
\mathcal{A}_{V}(I):=\left\{U(\varphi):\left.\varphi\right|_{I^{\prime}}=\operatorname{id}_{I^{\prime}}\right\}^{\prime \prime} \subset \mathcal{A}(\mathrm{I})
$$

where $I \in \mathcal{J}$ defines a subnet $\mathcal{A}_{V} \subset \mathcal{A}$.

Of course $V$ can be restricted to $\mathcal{H}_{\mathcal{A}_{V}}$ and the restriction of the subnet $\mathcal{A}_{V}$ onto this subspace is generated by the restriction of $V$ in the sense that local algebras are generated by the unitaries associated to local diffeomorphisms. Therefore by the irreducibility property this restriction of $V$ is irreducible. Since as we have already explained, irreducible representations are of the type $V_{(c, h)}$, the net $\left.\mathcal{A}_{V}\right|_{\mathcal{H}_{\mathcal{A}_{V}}}$ is a Virasoro net given by the representation $\left.V\right|_{\mathcal{H}_{\mathcal{A}_{V}}}$.

Finally, let us recall an important property of the Virasoro nets in connection with subnets. (See the proof in 22.)

Theorem 2.3 (Minimality of the Virasoro nets). A Virasoro net does not have any proper Möbius covariant subnet. 


\section{Uniqueness in case of the Virasoro nets}

In this section we prove the uniqueness of the $\operatorname{Diff}^{+}\left(S^{1}\right)$-action in the case of Virasoro nets. This result, of itself interest, will also provide an important step in the uniqueness proof for the general case of 4-regular nets that we shall discuss later.

Let $\tilde{V}$ be a positive energy projective representation of $\operatorname{Diff}^{+}\left(S^{1}\right)$ making $\mathcal{A}_{\mathrm{Vir}, c}$ diffeomorphism covariant in the sense of Definition 2.1. Of course in particular on the Möbius subgroup $\tilde{V}$ coincides with $V_{c}$.

Lemma 3.1. $\tilde{V}$ is irreducible.

Proof. As in the last subsection of the preliminaries, by the equation

$$
\mathcal{A}_{\tilde{V}}(I)=\left\{\tilde{V}(\gamma):\left.\gamma\right|_{I^{\prime}}=\mathrm{id}_{I^{\prime}}\right\}^{\prime \prime} \quad(\mathrm{I} \in \mathcal{J})
$$

we define a (Möbius covariant) subnet of $\mathcal{A}_{\mathrm{Vir}, c}$. Therefore, by the minimality (cited by us in the preliminaries as theorem 2.3) of the Virasoro net, taking account that $\tilde{V}$ cannot be trivial, we have that $\mathrm{B}\left(\mathcal{H}_{(c, 0)}\right)=\mathcal{A}_{\mathrm{Vir}, c}\left(S^{1}\right)=\mathcal{A}_{\tilde{V}}\left(S^{1}\right)=\{\tilde{V}\}^{\prime \prime}$.

As a consequence of lemma 3.1 and the fact ([4, Theorem A.1]) that the irreducible representations are exactly the Virasoro ones, $\tilde{V}$ is an irreducible Virasoro representation with lowest weight zero and central charge $\tilde{c}$ (with possibly $c \neq \tilde{c}$ ). We shall denote by $\left\{\tilde{L}_{n}: n \in \mathbb{Z}\right\}$ the resulting family of representing operators for the Virasoro algebra and by $\tilde{T}$ the corresponding stress-energy tensor.

On the Möb $\subset \operatorname{Diff}^{+}\left(S^{1}\right)$ subgroup $\tilde{V}$ coincides with $V_{c}$. Since the Möbius vector fields are exactly the ones for which the only nonzero Fourier coefficients are those associated to $-1,0,1$, we have that $\tilde{L}_{n}=L_{n}$ for $n=0,1,-1$. The notion of "finiteenergy" vectors (since $L_{0}=\tilde{L}_{0}$ ) is unambiguous, and any polynomial of the $L$ or $\tilde{L}$ operators is well-defined on $\mathcal{D}_{\text {fin }}$.

Lemma 3.2. There exists a complex number $\zeta$ such that for every $n \in \mathbb{Z}$ we have $\tilde{L}_{n} \Omega=\zeta L_{n} \Omega$.

Proof. From the theory of positive energy representations of the Virasoro algebra (see e.g. [14]) we know that every eigenvector of $L_{0}$ with eigenvalue 2 is proportional to the nonzero vector $L_{-2} \Omega$. Since $L_{0}=\tilde{L}_{0}$ and by the Virasoro algebra relations $\tilde{L}_{0}\left(\tilde{L}_{-2} \Omega\right)=$ $2 \tilde{L}_{-2} \Omega$, there must exist a complex number $\zeta$ such that $\tilde{L}_{-2} \Omega=\zeta L_{-2} \Omega$.

Both vectors $\tilde{L}_{-n} \Omega$ and $L_{-n} \Omega$ vanish if $n<2$, so we only have to show that $\tilde{L}_{-n} \Omega=$ $\zeta L_{-n} \Omega$ for every integer $n \geq 2$. We do this by induction. For $n=2$ the equality has been shown before. Now assume that $\tilde{L}_{-n} \Omega=\zeta L_{-n} \Omega$ for some $n \geq 2$. Then, recalling that $L_{-1}=\tilde{L}_{-1}$ and using the Virasoro algebra relations we find

$$
\begin{aligned}
(n-1) \tilde{L}_{-n-1} \Omega & =L_{-1} \tilde{L}_{-n} \Omega \\
& =\zeta L_{-1} L_{-n} \Omega \\
& =(n-1) \zeta L_{-n-1} \Omega,
\end{aligned}
$$

and the conclusion follows. 
We are now ready to state the main result of this section.

Theorem 3.3. $\tilde{V}$ as projective representation coincides with $V_{c}$. In other words, $\mathcal{A}_{\mathrm{Vir}, c}$ has a unique $\operatorname{Diff}^{+}\left(S^{1}\right)$ action which is compatible with the action of Möb determined by the net and its vacuum vector $\Omega$.

Proof. From Lemma 3.2 we find that $\tilde{T}(f) \Omega=\zeta T(f) \Omega$ for every real smooth function $f$. Now, if the support of $f$ is contained in an interval $I \in \mathcal{J}$ and $\Psi \in \mathcal{A}_{\mathrm{Vir}, c}\left(I^{\prime}\right) \Omega$, it follows from locality that $\tilde{T}(f) \Psi=\zeta T(f) \Psi$. But $\mathcal{A}_{\mathrm{Vir}, c}\left(I^{\prime}\right) \Omega$ contains a core for $L_{0}$ (see the appendix to [3]) and hence it is a common core for $T(f)$ and $\tilde{T}(f)$, see e.g. [1] or the next section. It follows that $\tilde{T}(f)=\zeta T(f)$ for every real smooth function $f$ on $S^{1}$ with nondense support and hence for every real function $f$ on $S^{1}$. In particular, since $\tilde{L}_{0}=L_{0}$ by assumption, we must have $\zeta=1$ and hence $\tilde{V}(\operatorname{Exp}(\mathrm{f}))=\mathrm{V}_{\mathrm{c}}(\operatorname{Exp}(\mathrm{f}))$ for every smooth real vector field $f$ on $S^{1}$. Our claim then follows because $\operatorname{Diff}^{+}\left(S^{1}\right)$ is generated by exponentials [23].

Corollary 3.4. Two Virasoro nets as Möbius covariant nets are isomorphic if and only if they have the same central charge.

\section{Stress-energy tensor and nonsmooth vector fields}

Suppose we have a positive energy representation of $\operatorname{Diff}^{+}\left(S^{1}\right)$. We would like to extend the representation to some transformations that are not smooth, but still "sufficiently regular". (Later we shall give more meaning to this.) For this purpose we shall take a not necessary smooth function $f: S^{1} \rightarrow \mathbb{R}$ (of which we think as a non-smooth vector field) and we will try to define a self-adjoint operator $T(f)$ by the closure of the naive formula $\sum_{n \in \mathbb{Z}} \hat{f}_{n} L_{n}$. (As it will be discussed, even if the representation is not irreducible, it gives rise to a corresponding representation $\left\{L_{n}: n \in \mathbb{Z}\right\}$ of the Virasoro algebra with all the properties listed in the preliminaries.)

Looking at the article of Goodman and Wallach [10], we can see that in fact everything works well with the definition of $T(f)$ even if $f$ is not smooth but for example if $\sum_{n \in \mathbb{Z}}\left|\hat{f}_{n}\right|(1+|n|)^{3}<\infty$. Unfortunately, for the uniqueness result we need to handle functions of less regularity. However, in the cited article essential self-adjointness is proved by using a result in the paper of Nelson [24]. Reading the work of Nelson, we can realize that what we really need is an $\epsilon$-independent bound on the norm of the commutator $\left[\sum_{n \in \mathbb{Z}} \hat{f}_{n} L_{n}, e^{-\epsilon L_{0}}\right]$ where $\epsilon>0$. This is what we shall establish in what follows here.

Throughout this section let $V$ be a positive energy representation of $\operatorname{Diff}^{+}\left(S^{1}\right)$. As $e^{i 2 \pi L_{0}}$ is a multiple of the identity, the nonnegative spectrum of $L_{0}$ contains eigenvalues only (at distances of integer numbers). So the linear span of the eigenvectors $\mathcal{D}_{\text {fin }}$ is still a dense subspace, which we shall still call the space of "finite-energy" vectors. In [21, Chapt. 1] T. Loke has shown that if the eigenspaces of $L_{0}$ are all finite dimensional than via infinitesimal generators in the same way as it was described in the preliminaries (but there only in the irreducible case) it gives rise to a representation of the Virasoro 
algebra with a certain value of the central charge. However, as it was pointed out in the appendix of [4], this condition can be dropped. So the construction with infinitesimal generators works and gives us in general a representation $\left\{L_{n}: n \in \mathbb{Z}\right\}$ of the Virasoro algebra with a certain value $c>0$ of the central charge satisfying all the properties already listed in subsection 2.2. Namely, $\mathcal{D}_{\text {fin }}$ is an invariant core for the closed operators $L_{n}(n \in \mathbb{Z})$, the adjoint operator $L_{n}^{*}$ equals to $L_{-n}$ for all $n \in \mathbb{Z}$ and on the common invariant core of the finite-energy vectors these operators satisfy the Virasoro algebra relations.

Although equation (2.8) on page no. 308 in the article of Goodman and Wallach [10] is stated for the irreducible case, the value of the lowest weight is not involved at all and in fact after a close look it is rather evident that as a consequence we have in general the following:

Lemma 4.1. There exists a constant $r>0$ independent from $k, v_{k}, n$ (but dependent on the value of the central charge $c$ ) such that

$$
\left.\left\|L_{n} v_{k}\right\|^{2} \leq r^{2}\left(k^{2}+k|n|^{2}+|n|^{3}\right)\right)\left\|v_{k}\right\|^{2}
$$

where $v_{k}$ is an eigenvector of $L_{0}$ with eigenvalue $k$ and $n \in \mathbb{Z}$.

It is clear therefore, that $\mathcal{D}\left(L_{0}\right)$, the domain of $L_{0}$, is included in the domain of $L_{n}$, and if $v \in \mathcal{D}\left(L_{0}\right)$ then by using that $\sqrt{1+|n|^{3}} \leq\left(1+|n|^{\frac{3}{2}}\right)$

$$
\left\|L_{n} v\right\| \leq r\left(1+|n|^{\frac{3}{2}}\right)\left\|\left(\mathbb{1}+L_{0}\right) v\right\|
$$

which is why any core for $L_{0}$ is a core for $L_{n}$. (And in particular, as we have already stated, the finite-energy space is so.) Related "energy-bounds" can be found in [1]. The above estimate has the following consequence.

Proposition 4.2. If $a_{n} \in \mathbb{C}(n \in \mathbb{Z})$ is such that $\sum_{n \in \mathbb{Z}}\left|a_{n}\right|\left(1+|n|^{\frac{3}{2}}\right)<\infty$ then

(i) the operator $A=\sum_{n \in \mathbb{Z}} a_{n} L_{n}$ with domain $\mathcal{D}\left(L_{0}\right)$ is well defined, (i.e. the sum strongly converges on the domain);

(ii) if $v \in \mathcal{D}\left(L_{0}\right)$, then as $N \rightarrow \infty$ the sum

$$
\sum_{k \in \operatorname{Sp}\left(L_{0}\right), k \leq N} A v_{k} \rightarrow A v
$$

strongly, where the vector $v_{k}$ is the component of $v$ in the eigenspace of $L_{0}$ associated to the value $k \in S p\left(L_{0}\right)$,

(iii) $A^{*}$ is an extension of the operator $A^{+}:=\sum_{n \in \mathbb{Z}} \bar{a}_{-n} L_{n}$. (This again is understood as an operator with domain $\mathcal{D}\left(L_{0}\right)$.) 
Proof. Since the sum

$$
\sum_{n \in \mathbb{Z}}\left\|a_{n} L_{n} v\right\| \leq r\left(\sum_{n \in \mathbb{Z}}\left|a_{n}\right|\left(1+|n|^{\frac{3}{2}}\right)\right)\left\|\left(\mathbb{1}+L_{0}\right) v\right\|<\infty
$$

claim (i) holds. Claim (ii) follows from the same estimate and the fact that

$$
\left(\mathbb{1}+L_{0}\right)\left(v-\sum_{k \in \operatorname{Sp}\left(L_{0}\right), k \leq N} v_{k}\right) \rightarrow 0
$$

as $N$ tends to $\infty$. Finally, the last claim follows, since for all $n$ integer $L_{n}^{*}=L_{-n}$.

We now consider, for every $\epsilon>0$, the operator $R_{n, \epsilon}=\left[L_{n}, e^{-\epsilon L_{0}}\right]$ which is at least densely defined for every $n \in \mathbb{Z}$, since its domain surely contains the subspace $\mathcal{D}\left(L_{0}\right)$. The following proposition gives an estimate on the norm of this commutator which is independent of $\epsilon$.

Proposition 4.3. There exists a constant $q>0$ independent of $\epsilon$ and $n$ such that $\left\|R_{n, \epsilon}\right\|^{2}=\left\|\left[L_{n}, e^{-\epsilon L_{0}}\right]\right\|^{2} \leq q|n|^{3}$.

Proof. For $n=0$ the statement is trivially true as $L_{0}$ commutes with any bounded function of itself. Since $L_{n}^{*}=L_{-n}$ and $e^{-\epsilon L_{0}}$ is self-adjoint it follows that $R_{n, \epsilon} \subset-R_{-n, \epsilon}^{*}$, it suffices to demonstrate the statement for negative values of $n$, and, since it also shows that $R_{n, \epsilon}$ is closable, it is enough to verify that $\left\|R_{n, \epsilon} v\right\|^{2} \leq q|n|^{3}\|v\|^{2}$ whenever $v \in \mathcal{D}_{\text {fin }}$.

Let therefore be $n<0, v \in \mathcal{D}_{\text {fin }}$ and for every $k \in \operatorname{Sp}\left(L_{0}\right)$ let $v_{k}$ be again the component of the vector $v$ in the eigenspace of $L_{0}$ associated to the eigenvalue $k$. To not to get confused about positive and negative constants, in the calculations we shall use the positive $m:=-n$ rather than the negative $n$. Now since $L_{n}$ raises the eigenvalue of $L_{0}$ by $m$, we have that for $k \in \operatorname{Sp}\left(L_{0}\right)$

$$
R_{n, \epsilon} v_{k}=\left[L_{n}, e^{-\epsilon L_{0}}\right] v_{k}=\left(e^{-\epsilon k}-e^{-\epsilon(k+m)}\right) L_{n} v_{k} .
$$

The mapping $f_{m}: \epsilon \mapsto e^{-\epsilon k}-e^{-\epsilon(k+m)}$ is a positive smooth function on $\mathbb{R}^{+}$which goes to zero both when $\epsilon \rightarrow 0$ and when $\epsilon \rightarrow \infty$. Therefore $f_{m}$ has a maximum on $\mathbb{R}^{+}$. Now the only solution of the equation $f_{m}^{\prime}(\epsilon)=0$ is $\epsilon_{m}=-(1 / m) \ln (k /(k+m))$. This, together with the mentioned facts gives that

$$
\sup _{\epsilon \in \mathbb{R}^{+}}\left|f_{m}(\epsilon)\right|^{2}=f_{m}\left(\epsilon_{m}\right)^{2}=\left(\frac{k}{k+m}\right)^{\frac{2 k}{m}}\left(\frac{m}{k+m}\right)^{2} \leq\left(\frac{m}{k+m}\right)^{2}
$$

We can now return to the question of the norm of the commutator. Equation (16) shows that the vectors $R_{n, \epsilon} v_{k}\left(k \in \operatorname{Sp}\left(L_{0}\right)\right)$ are in particular pairwise orthogonal. Using 
this and the fact that only for finitely many values of $k$ the vector $v_{k} \neq 0$ we find

$$
\begin{aligned}
\left\|R_{n, \epsilon} v\right\|^{2} & =\left\|R_{n, \epsilon} \sum_{k \in \operatorname{Sp}\left(L_{0}\right)} v_{k}\right\|^{2}=\sum_{k \in \operatorname{Sp}\left(L_{0}\right)}\left\|R_{n, \epsilon} v_{k}\right\|^{2}=\sum_{k \in \operatorname{Sp}\left(L_{0}\right)}\left|f_{m}(\epsilon)\right|^{2}\left\|L_{n} v_{k}\right\|^{2} \\
& \leq \sum_{k \in \operatorname{Sp}\left(L_{0}\right)} \sup _{\epsilon \in \mathbb{R}^{+}}\left\{\left|f_{m}(\epsilon)\right|^{2}\right\}\left\|L_{n} v_{k}\right\|^{2} \\
& \leq \sum_{k \in \operatorname{Sp}\left(L_{0}\right)}\left(\frac{m}{k+m}\right)^{2} r^{2}\left(k^{2}+k m^{2}+m^{3}\right)\left\|v_{k}\right\|^{2} \\
& \leq \sum_{k \in \operatorname{Sp}\left(L_{0}\right)} r^{2}\left(m^{2}+m^{3}+m^{3}\right)\left\|v_{k}\right\|^{2} \\
& \leq 3 r^{2}|n|^{3}\|v\|^{2}
\end{aligned}
$$

where we have used the inequality (17) and the constant $r$ is the one coming from lemma 4.1 in estimating the norm square of $L_{n} v_{k}$.

Theorem 4.4. If $a_{n} \in \mathbb{C}(n \in \mathbb{Z})$ is such that $\sum_{n \in \mathbb{Z}}\left|a_{n}\right||n|^{\frac{3}{2}}<\infty$ then $A$ is closable and $\bar{A}=\left(A^{+}\right)^{*}$, where $A=\sum_{n \in \mathbb{Z}} a_{n} L_{n}$ and $A^{+}=\sum_{n \in \mathbb{Z}} \bar{a}_{-n} L_{n}$ considered as operators on the domain $\mathcal{D}_{\text {fin }}$. In particular, if $a_{n}=\overline{a_{-n}}$ for all $n \in \mathbb{Z}$, then $A$ is essentially self-adjoint on $\mathcal{D}_{\text {fin }}$.

Proof. Let us first note, that because of Proposition 4.2, claim (iii) the operator $A$ both with domain $\mathcal{D}\left(L_{0}\right)$ and $\mathcal{D}_{\text {fin }}$ is closable, since the domain of its adjoint surely contains $\mathcal{D}\left(L_{0}\right)$ and that because of proposition 4.2, claim (ii) $\overline{\left.A\right|_{\mathcal{D}_{\text {fin }}}}=\overline{\left.A\right|_{\mathcal{D}\left(L_{0}\right)}}$. Therefore from now on we shall think of $A$ as an operator with domain $\mathcal{D}\left(L_{0}\right)$, since in any case it does not change neither its closure nor its adjoint. (Of course the same applies to the operator $A^{+}$.) Further, if $\epsilon>0$, then the domain of the operator $R_{A, \epsilon}=\left[A, e^{-\epsilon L_{0}}\right]$ is the whole $\mathcal{D}\left(L_{0}\right)$ and we have that $R_{A, \epsilon} \subset-R_{A^{+}, \epsilon}^{*}$ where $R_{A^{+}, \epsilon}=\left[A^{+}, e^{-\epsilon L_{0}}\right]$. By using Proposition 4.3 with the constant $q$ provided by it and the condition on the sequence $a_{n}(n \in \mathbb{Z})$,

$$
\sum_{n \in \mathbb{Z}}\left\|a_{n} R_{n, \epsilon}\right\| \leq \sum_{n \in \mathbb{Z}}\left|a_{n}\right| q^{\frac{1}{2}}|n|^{\frac{3}{2}}<\infty .
$$

Since $R_{A, \epsilon}=\sum_{n \in \mathbb{Z}} a_{n} R_{n, \epsilon}$ on $\mathcal{D}\left(L_{0}\right)$, this means that $\left\|R_{A, \epsilon}\right\|$ is bounded by a constant independent of $\epsilon$. Obviously, the same is true for $\left\|R_{A^{+}, \epsilon}\right\|$.

If $v_{k}$ is an eigenvector of $L_{0}$ with eigenvalue $k \geq 0$ then, as $\epsilon$ tends to zero,

$$
R_{A^{+}, \epsilon} v_{k}=\left(e^{-\epsilon k} \mathbb{1}-e^{-\epsilon L_{0}}\right) A^{+} v_{k} \rightarrow 0 .
$$

Thus the operators $R_{A^{+}, \epsilon}$ on $\mathcal{D}_{\text {fin }}$ strongly converge to zero. Then since their norm is bounded by a constant independent of $\epsilon$, as $\epsilon \rightarrow 0$, the everywhere defined bounded operators $R_{A, \epsilon}^{*}=-\overline{R_{A^{+}, \epsilon}}$ converge strongly to zero.

From here the proof of the theorem continues exactly as in [24, but for selfcontainment let us revise the concluding argument. Suppose $x$ is a vector in the domain of $A^{*}$. Then, since $e^{-\epsilon L_{0}} x \in \mathcal{D}\left(L_{0}\right) \subset \mathcal{D}\left(A^{+}\right)$, we have that

$$
A^{+} e^{-\epsilon L_{0}} x=A^{*} e^{-\epsilon L_{0}} x=e^{-\epsilon L_{0}} A^{*} x-R_{A, \epsilon}^{*} x .
$$


As $\epsilon \rightarrow 0$ of course $e^{-\epsilon L_{0}} x \rightarrow x$, but now Equation (21) shows that also $A^{+} e^{-\epsilon L_{0}} x \rightarrow A^{*} x$ strongly. Therefore $A^{*}=\overline{A^{+}}$.

With this we have proved the main theorem of this section. The result ensures, that if the continuous function $f: S^{1} \rightarrow \mathbb{R}$ with Fourier coefficients $\hat{f}_{n}(n \in \mathbb{Z})$ is such, that the norm

$$
\|f\|_{\frac{3}{2}}=\sum_{n \in \mathbb{Z}}\left|\hat{f}_{n}\right|\left(1+|n|^{\frac{3}{2}}\right)
$$

is finite, then $\sum_{n \in \mathbb{Z}} \hat{f}_{n} L_{n}$ is an essentially self-adjoint operator on $\mathcal{D}_{\text {fin }}$. As in the case of smooth functions, we will denote by $T(f)$ the corresponding self-adjoint operator obtained by taking closure. We continue by investigating the continuity property of the stress-energy tensor $T$.

Proposition 4.5. For every continuous real function $f$ on $S^{1}$ of finite $\|\cdot\|_{\frac{3}{2}}$ norm and for every $v \in \mathcal{D}\left(L_{0}\right)$ we have

$$
\|T(f) v\| \leq r\|f\|_{\frac{3}{2}}\left\|\left(\mathbb{1}+L_{0}\right) v\right\|
$$

where $r$ is the positive constant appearing in Lemma 4.1. Moreover, if $f$ and $f_{n}(n \in \mathbb{N})$ are continuous real functions on $S^{1}$ of finite $\|\cdot\|_{\frac{3}{2}}$ norm, and $\left\|f_{n}-f\right\|_{\frac{3}{2}}$ converges to zero as $n$ tends to $\infty$, then $T\left(f_{n}\right) \rightarrow T(f)$ in the strong resolvent sense. In particular, $e^{i T\left(f_{n}\right)} \rightarrow e^{i T(f)}$ strongly.

Proof. The claimed inequality is an immediate consequence of the inequality in Eq. (14) and the definition of the $\|\cdot\|_{\frac{3}{2}}$ norm. Now by this estimate for every $v \in \mathcal{D}\left(L_{0}\right)$ we have that $T\left(f_{n}\right) v$ converges to $T(f) v$. Since $\mathcal{D}\left(L_{0}\right)$ is a common core for these self-adjoint operators, the conclusion follows (see e.g. [25, Sect. VIII.7]).

In the next section we shall need to determine the geometrical properties of the adjoint action of $e^{i T(f)}$ for a certain $f$ nonsmooth vector field. If $f$ was smooth, we would know what the unitary $e^{i T(f)}$ "does" since it is the operator associated by the representation to the diffeomorphism $\operatorname{Exp}(f)$. Thanks to the last proposition, to obtain information in the case when $f$ is not smooth, all we will have to do is to approximate it with smooth ones in an appropriate way. As it will be clear later, the following lemma shows that for our purposes the smooth vector fields are "many enough".

Lemma 4.6. Let $I \subset S^{1}, I \neq \emptyset$ be an open interval (or even the whole circle). If $f$ is a real continuous function of finite $\|\cdot\|_{\frac{3}{2}}$ norm with support contained in $I$, then there exists a sequence $f_{k},(k=1,2, .$.$) of real smooth functions with support still in I$ such that $\lim _{k \rightarrow \infty}\left\|f_{k}-f\right\|_{\frac{3}{2}}=0$.

Proof. The proof follows standard arguments relying on convolution with smooth functions. Let $\varphi_{k}(k=1,2, .$.$) be a sequence of positive smooth functions on S^{1}$ with support shrinking to the point $1 \in S^{1}$ such that for all $k \in \mathbb{N}$

$$
\frac{1}{2 \pi} \int_{0}^{2 \pi} \varphi_{k}\left(e^{i \alpha}\right) d \alpha=1
$$


Then for $k$ large enough the convolution $\varphi_{k} * f$ is a smooth real function with support in $I$. Moreover we have

$$
\left\|\varphi_{k} * f-f\right\|_{\frac{3}{2}} \leq \sum_{n \in \mathbb{Z}}\left|\left(\left(\hat{\varphi}_{k}\right)_{n}-1\right) \hat{f}_{n}\right|\left(1+|n|^{\frac{3}{2}}\right)
$$

where the left-hand side goes to zero when $k \rightarrow \infty$ since $\left|\left(\hat{\varphi}_{k}\right)_{n}\right| \leq 1$ and $\lim _{k \rightarrow \infty}\left(\hat{\varphi}_{k}\right)_{n}=$ 1 .

\section{$5 \quad$ Uniqueness in case of 4-regularity}

Suppose $(\mathcal{A}, U)$ is a Möbius covariant net on the circle which is also diffeomorphism covariant with the representation $V$ of $\operatorname{Diff}^{+}\left(S^{1}\right)$. Further, suppose that there exists another positive energy representation $\tilde{V}$ of $\operatorname{Diff}^{+}\left(S^{1}\right)$ which also makes $(\mathcal{A}, U)$ diffeomorphism covariant in the sense of Definition 2.1. The representations $V$ and $\tilde{V}$, as before, via infinitesimal generators, give rise the representations $L$ and $\tilde{L}$ of the Virasoro algebra (with possibly different values of the central charge). The corresponding stressenergy tensor fields we shall denote by $T$ and $\tilde{T}$. As the two projective representations coincide on the Möbius subgroup, $L_{n}=\tilde{L}_{n}$ for $n=-1,0,1$ or to put it in another way, $T(g)=\tilde{T}(g)$ whenever $g$ is a Möbius vector field.

Considering the two representations we can define two Möbius covariant subnets: the subnet $\mathcal{A}_{V}$ defined for every open proper arc $I \subset S^{1}$ by

$$
\mathcal{A}_{V}(I)=\left\{V(\varphi)|\varphi|_{I^{\prime}}=\mathrm{id}_{I^{\prime}}\right\} \subset \mathcal{A}(\mathrm{I})
$$

and the subnet $\mathcal{A}_{\tilde{V}}$ defined similarly but with the representation $V$ replaced by $\tilde{V}$. The restriction of the subnet $\mathcal{A}_{V}$ onto the closed linear subspace $\overline{\mathcal{A}_{V} \Omega}$, as it has been cited from 4 several times by now, is a Virasoro net for a certain value of central charge $c$ and so it will be denoted by $\mathcal{A}_{\text {Vir }}$. In the same way the restriction of $\mathcal{A}_{\tilde{V}}$ onto $\overline{\mathcal{A}_{\tilde{V}} \Omega}$ is another Virasoro net (with the possibly different value of central charge $\tilde{c}$ ) and will be denoted by $\mathcal{A}_{\tilde{\text { Vir }}}$. But a Virasoro net is a minimal net, and this gives a very strong restriction on the possible ways the two subnets $\mathcal{A}_{V}$ and $\mathcal{A}_{\tilde{V}}$ can "differ".

Proposition 5.1. If $\mathcal{A}_{V}$ and $\mathcal{A}_{\tilde{V}}$ as subnets are not equal, then for all $I \subset S^{1}$ open proper arc $\mathcal{A}_{V}(I) \cap \mathcal{A}_{\tilde{V}}(I)=\mathbb{C} \mathbb{1}$.

Proof. Suppose that $\mathcal{A}_{V}(I) \cap \mathcal{A}_{\tilde{V}}(I)$ is nontrivial for a given (and hence, by Möbius covariance, for all) $I \in \mathcal{J}$. Then the subnet $I \mapsto \mathcal{A}_{V}(I) \cap \mathcal{A}_{\tilde{V}}(I) \subset \mathcal{A}(I)$ when restricted to $\overline{\mathcal{A}_{V} \Omega}$ is a Möbius covariant subnet of $\mathcal{A}_{\mathrm{Vir}}$, therefore by minimality (cited by us as Theorem [2.3) it must coincide with $\mathcal{A}_{\text {Vir }}$. On the other hand, for an open proper arc $I$ the restriction map from $\mathcal{A}_{V}(I)$ to $\mathcal{A}_{\mathrm{Vir}}(I)$ is an isomorphism. So we have that $\mathcal{A}_{V}(I) \cap \mathcal{A}_{\tilde{V}}(I)$ coincides with $\mathcal{A}_{V}(I)$ for every $I \in \mathcal{J}$. But of course by interchanging $V$ and $\tilde{V}$, it must also coincide with $\mathcal{A}_{\tilde{V}}(I)$ for every $I \in \mathcal{J}$ and this concludes the proof. 
Proposition 5.2. If $\mathcal{A}_{V}$ and $\mathcal{A}_{\tilde{V}}$ as subnets are equal then so are $V$ and $\tilde{V}$ as projective representation; i.e. $\operatorname{Ad}(\mathrm{V}(\varphi))=\operatorname{Ad}(\tilde{\mathrm{V}}(\varphi))$ for all $\varphi \in \operatorname{Diff}^{+}\left(S^{1}\right)$.

Proof. By the condition of the proposition the representation $\tilde{V}$ can be restricted onto $\overline{\mathcal{A}_{V} \Omega}$ and this gives a positive energy representation of $\operatorname{Diff}^{+}\left(S^{1}\right)$, which is compatible with the Virasoro net $\mathcal{A}_{\text {Vir }}$ (as Möbius covariant net). Hence, by the uniqueness result for the Virasoro nets (Theorem 3.3) it must be equal (as a projective representation) with the restriction of $V$ onto the same subspace. But if $\varphi$ is a diffeomorphism "localized" in the open proper arc $I \subset S^{1}$, that is, $\left.\varphi\right|_{I^{\prime}}=\mathrm{id}_{\mathrm{I}^{\prime}}$, then - since $V(\varphi), \tilde{V}(\varphi) \in \mathcal{A}_{V}(I)$, and the restriction map from $\mathcal{A}_{V}(I)$ to $\mathcal{A}_{\mathrm{Vir}}(I)$ is an isomorphism — the operator $V(\varphi)^{*} \tilde{V}(\varphi)$ must be a multiple of the identity. This is enough for the equality, since Diff ${ }^{+}\left(S^{1}\right)$ is generated by localized diffeomorphisms.

Thus, by the previous two propositions if the local intersections of the subnets $\mathcal{A}_{V}$ and $\mathcal{A}_{\tilde{V}}$ are not trivial, then we have that $V$ and $\tilde{V}$, as projective representations, are equal. We know that the intersection of the two algebras $\mathcal{A}_{V}\left(S^{1}\right)$ and $\mathcal{A}_{\tilde{V}}\left(S^{1}\right)$ cannot be trivial: it contains the unitaries associated to Möbius transformations, for example. Unfortunately, there is no Möbius transformation - apart from the identity — that would be local. However, we can construct local transformations that are piecewise Möbius. Naturally, they will not be smooth, but, as we shall see it, by choosing the parameters rightly, we can achieve once differentiability, with discontinuities ("jumps") appearing at the endpoints of the pieces only in the second derivative. We have essentially three things to do:

(i) we have to construct such a $\zeta$ piecewise Möbius transformation,

(i) we must show, that although $\zeta$ is not smooth, it is sufficiently regular so that the expressions $V(\zeta)$ and $\tilde{V}(\zeta)$ are meaningful, and finally,

(ii) we must show that the adjoint action of these unitaries on the algebras corresponding to some pieces is completely determined (since the geometrical part of this action on each piece is Möbius), and we must investigate that under what condition on the net it implies that the two unitaries are in fact multiples of each other.

We begin with the construction of a piecewise Möbius transformation. For a $z \in S^{1}$ let $I_{(z, i z)} \subset S^{1}$ be the open quarter-arc with endpoints $z$ and $i z$. The real Möbius vector field $g_{1}$ given by the formula

$$
g_{1}(z)=(i-1) z+2-(i+1) z^{-1}
$$

is zero in the two points $1, i \in S^{1}$. Hence by setting $g_{p}(p=1, i,-1,-i)$ to be the real Möbius vector field determined by the equation

$$
g_{p}(p z)=p^{2} g_{1}(z)
$$


the map

$$
z \mapsto\left\{\begin{array}{cc}
g_{1}(z) & \text { if } z \in I_{(1, i)} \\
g_{i}(z) & \text { if } z \in I_{(i,-1)} \\
g_{-1}(z) & \text { if } z \in I_{(-1,-i)} \\
g_{-i}(z) & \text { if } z \in I_{(-i, 1)}
\end{array}\right.
$$

defines a unique continuous function $f: S^{1} \rightarrow \mathbb{R}$. We shall think of this function as a nonsmooth vector field. It has four points at which it is zero: the points $1, i,-1$ and $-i$. On each of the four quarter-arc between these points it coincides with a Möbius vector field (of course on each arc with a different one).

Lemma 5.3. $\|f\|_{\frac{3}{2}}<\infty$

Proof. By direct calculation not only $f$, but also its derivative is continuous. Its second derivative is of course still smooth on each of the four open intervals, and at the endpoints it has only finite "jumps". Therefore it is a function of bounded variation, and hence there is a constant $M>0$ such that the absolut value of its Fourier coefficient associated to any integer $n$ is bounded by $\frac{M}{|n|}$ (see [15, Sect. I.4]) which in turn implies that $\left|\hat{f}_{n}\right| \leq \frac{M}{|n|^{3}}$ for all $n \in \mathbb{Z}$.

This means that we can consider the self-adjoint operators $T(f)$ and $\tilde{T}(f)$. By construction, $T(f)$ is affiliated to $\mathcal{A}_{V}\left(S^{1}\right)$ and $\tilde{T}(f)$ is affiliated to $\mathcal{A}_{\tilde{V}}\left(S^{1}\right)$.

Proposition 5.4. For all $t \in \mathbb{R}$ the adjoint actions of $e^{i t T(f)}$ and $e^{i t \tilde{T}(f)}$ restricted to the algebra $\mathcal{A}\left(I_{(p, i p)}\right)$ coincide with that of $e^{i t T\left(g_{p}\right)}=e^{i t \tilde{T}\left(g_{p}\right)}$, where $p=1, i,-1,-i$.

Proof. The continuous real function $f-g_{p}$ is zero on $I_{(p, i p)}$. Since its $\|\cdot\|_{\frac{3}{2}}$ norm is finite, we can consider the operator $T\left(f-g_{p}\right)$ which then by Lemma 4.6 and Proposition 4.5 is affiliated to $\mathcal{A}\left(I_{(p, i p)}^{\prime}\right)$. On the common core of the finite-energy vectors

$$
T(f)=T\left(f-g_{p}\right)+T\left(g_{p}\right) .
$$

Then, since $T\left(f-g_{p}\right)$ is affiliated to the commutant of $\mathcal{A}\left(I_{(p, i p)}\right)$ and

$$
\operatorname{Ad}\left(\mathrm{e}^{\mathrm{itT}\left(\mathrm{g}_{\mathrm{p}}\right)}\right)\left(\mathcal{A}\left(\mathrm{I}_{(\mathrm{p}, \mathrm{ip})}\right)=\mathcal{A}\left(\mathrm{I}_{(\mathrm{p}, \mathrm{ip})}\right)\right.
$$

for all $t \in \mathbb{R}$, by a simple use of the Trotter product-formula (e.g. 25, Theorem VIII.31]) we have the part of the proposition concerning the adjoint action of $e^{i t T(f)}$. Similar argument justifies the assertions for $e^{i t \tilde{T}(f)}$.

This means that on the algebra associated to the four open quarter-arc the adjoint actions of $e^{i t T(f)}$ and $e^{i t \tilde{T}(f)}$ coincide. Hence if $\mathcal{A}$ is at least 4-regular, the unitary $e^{i t T(f)} e^{-i t \tilde{T}(f)}$ must be a multiple of the identity. It follows that $e^{i t T(f)} e^{-i t T\left(g_{1}\right)} \in \mathcal{A}_{V}\left(S^{1}\right)$ and $e^{i t \tilde{T}(f)} e^{-i t \tilde{T}\left(g_{1}\right)} \in \mathcal{A}_{\tilde{V}}\left(S^{1}\right)$ are multiples of each other and — since they act trivially on $\mathcal{A}\left(I_{(1, i)}\right)$ - that they belong to the local intersection $\mathcal{A}_{V}\left(I_{(1, i)}^{\prime}\right) \cap \mathcal{A}_{\tilde{V}}\left(I_{(1, i)}^{\prime}\right)$ for all real $t$. But of course they cannot be just multiples of the identity: for example because 
$T(f) \Omega$ is a nonzero vector (the real $f$ is not a Möbius vector field, so it cannot have zero Fourier coefficients associated to all values of $n<-1$ ) which is orthogonal to $\Omega$. Then, by Prop. 5.1 and Prop. 5.2 we can conclude that $V$ and $\tilde{V}$, as projective representations are equal.

Thus we have proved that

Theorem 5.5. Let $(\mathcal{A}, U)$ be an at least 4-regular diffeomorphism covariant net on the circle. Then there is a unique projective representation $V$ of $\operatorname{Diff}^{+}\left(S^{1}\right)$ which makes $(\mathcal{A}, U)$ diffeomorphism covariant in the sense of Definition 2.1.

Let us formulate now some important consequences of the fact that the whole representation $V$ must already be encoded in the Möbius covariant net (with its given representation of the Möbius group, or equivalently, with its given vacuum vector). Remember that a positive energy representation of $\operatorname{Diff}^{+}\left(S^{1}\right)$ always gives rise to a representation of the Virasoro algebra (see the discussion in the beginning of Section 4), so in particular it always has a central charge.

Corollary 5.6. Let $(\mathcal{A}, U)$ be a 4-regular net with the representation $V$ of $\operatorname{Diff}^{+}\left(S^{1}\right)$ making it diffeomorphism covariant. Then the representation class of $V$, and in particular its central charge $c>0$ is an invariant of the Möbius covariant net $(\mathcal{A}, U)$.

Another interesting thing to note here is the model-independent proof for the commutation between internal symmetries and diffeomorphism symmetry.

Definition 5.7. A unitary $W$ on the Hilbert space $\mathcal{H}_{\mathcal{A}}$ is called an (unbroken) internal symmetry of the net $(\mathcal{A}, U)$ if for every $I \in \mathcal{J}$

$$
W \mathcal{A}(I) W^{*}=\mathcal{A}(I)
$$

and $W \Omega=\Omega$ where $\Omega$ is the vacuum vector of $(\mathcal{A}, U)$.

By our uniqueness theorem we can state the following conclusion.

Corollary 5.8. Let $W$ be an internal symmetry of the net $(\mathcal{A}, U)$ having diffeomorphism symmetry. If $\mathcal{A}$ is at least 4-regular, than the unique representation $V$ of $\operatorname{Diff}^{+}\left(S^{1}\right)$ making the net diffeomorphism covariant must commute with $W$.

Proof. Since $W$ commutes with the representation $U$ (see [9]) the projective representation $W V W^{*}$ of $\operatorname{Diff}^{+}\left(S^{1}\right)$ still makes the net $(\mathcal{A}, U)$ diffeomorphism covariant. Hence by Theorem [5.5 it must coincide with $V$. It follows that for every $\gamma \in \operatorname{Diff}^{+}\left(S^{1}\right)$ the unitary $W V(\gamma) W^{*} V(\gamma)^{*}$ is a multiple $\lambda(\gamma)$ of the identity, and in fact it turns out that the complex valued function $\gamma \mapsto \lambda(\gamma)$ is a character of the group $\operatorname{Diff}^{+}\left(S^{1}\right)$. But the latter is a simple noncommutative group (see e.g. [23]), and hence $\lambda$ is trivial. 


\section{Infinite tensor products and nets admitting no diffeomorphism symmetry}

In this section we shall use our uniqueness results to exhibit a class of Möbius covariant nets on $S^{1}$ that definitely do not have a diffeomorphism symmetry.

Let $\left(\mathcal{A}_{n}, U_{n}\right), n=1,2, \ldots$ be a sequence of Möbius covariant nets on $S^{1}$ and let $\Omega_{n}$, $n=1,2, \ldots$ be the corresponding sequence of vacuum vectors. We can define the infinite tensor product net

$$
\mathcal{A} \equiv \bigotimes_{n} \mathcal{A}_{n}
$$

on the (separable) infinite tensor product Hilbert space

$$
\mathcal{H}_{\mathcal{A}}:=\bigotimes_{n}^{\left(\Omega_{n}\right)} \mathcal{H}_{\mathcal{A}_{n}}
$$

by

$$
\mathcal{A}(I):=\bigotimes_{n} \mathcal{A}_{n}(I)
$$

cf. [29]. It is fairly easy to show that $\mathcal{A}$ together with the representation $\bigotimes_{n} U_{n}$ is a Möbius covariant net on $S^{1}$ which is strongly additive (resp. n-regular) when each net $\mathcal{A}_{n}, n=1,2 \ldots$ is strongly additive (resp. n-regular).

We shall need the following proposition which is of interest of its own.

Proposition 6.1. Let, $\mathcal{A}, \mathcal{B}$, two 4-regular Möbius covariant nets on $S^{1}$. If $\mathcal{A}$ and $\mathcal{A} \otimes \mathcal{B}$ are diffeomorphism covariant and $V_{\mathcal{A}}, V_{\mathcal{A} \otimes \mathcal{B}}$ are the corresponding representations of $\operatorname{Diff}^{+}\left(S^{1}\right)$, then $\mathcal{B}$ is diffeomorphism covariant with a representation $V_{\mathcal{B}}$ satisfying $V_{\mathcal{A}} \otimes V_{\mathcal{B}}=V_{\mathcal{A} \otimes \mathcal{B}}$

Proof. Let us consider the Möbius covariant net $\mathcal{A} \otimes \mathcal{A} \otimes \mathcal{B}$. By assumption it is a 4-regular diffeomorphism covariant net on $S^{1}$ and the corresponding representation of $\operatorname{Diff}^{+}\left(S^{1}\right)$ is given by $V:=V_{\mathcal{A}} \otimes V_{\mathcal{A} \otimes \mathcal{B}}$. Let $F$ be the unitary operator on $\mathcal{H}_{\mathcal{A}} \otimes \mathcal{H}_{\mathcal{A}} \otimes \mathcal{H}_{\mathcal{B}}$ which flips the first two components of the tensor product. It is easy to see that $F$ is an internal symmetry of the net $\mathcal{A} \otimes \mathcal{A} \otimes \mathcal{B}$ and hence, by Corollary 5.8, it must commute with $V$. Since, for every $C \in \mathrm{B}\left(\mathcal{H}_{\mathcal{A}}\right), \gamma \in \operatorname{Diff}^{+}\left(S^{1}\right)$, we have

$$
V(\gamma)(C \otimes \mathbb{1} \otimes \mathbb{1}) V(\gamma)^{*}=\left(V_{\mathcal{A}}(\gamma) C V_{\mathcal{A}}(\gamma)^{*}\right) \otimes \mathbb{1} \otimes \mathbb{1}
$$

we find

$$
\begin{aligned}
V(\gamma)(\mathbb{1} \otimes C \otimes \mathbb{1}) V(\gamma)^{*} & =F V(\gamma) F^{*}(\mathbb{1} \otimes C \otimes \mathbb{1}) F V(\gamma)^{*} F^{*} \\
& =F V(\gamma)(C \otimes \mathbb{1} \otimes \mathbb{1}) V(\gamma)^{*} F^{*} \\
& =F\left(\left(V_{\mathcal{A}}(\gamma) C V_{\mathcal{A}}(\gamma)^{*}\right) \otimes \mathbb{1} \otimes \mathbb{1}\right) F^{*} \\
& =\mathbb{1} \otimes\left(V_{\mathcal{A}}(\gamma) C V_{\mathcal{A}}(\gamma)^{*}\right) \otimes \mathbb{1}
\end{aligned}
$$


It follows that, for every $\gamma \in \operatorname{Diff}^{+}\left(S^{1}\right)$,

$$
V(\gamma)\left(V_{\mathcal{A}}(\gamma) \otimes V_{\mathcal{A}}(\gamma) \otimes \mathbb{1}\right)^{*} \in\left(\mathrm{B}\left(\mathcal{H}_{\mathcal{A}}\right) \otimes \mathrm{B}\left(\mathcal{H}_{\mathcal{A}}\right) \otimes \mathbb{1}\right)^{\prime}=\mathbb{1} \otimes \mathbb{1} \otimes \mathrm{B}\left(\mathcal{H}_{\mathcal{B}}\right)
$$

and hence that there is a projective unitary representation $V_{\mathcal{B}}$ of $\operatorname{Diff}^{+}\left(S^{1}\right)$ on $\mathcal{H}_{\mathcal{B}}$ such that

$$
V=V_{\mathcal{A}} \otimes V_{\mathcal{A}} \otimes V_{\mathcal{B}}
$$

Then, the conclusion easily follows.

We are now ready to state the main result of this section.

Theorem 6.2. Let $\mathcal{A}_{n}, n \in \mathbb{N}$ be a sequence of 4-regular diffeomorphism covariant nets on $S^{1}$. Then the infinite tensor product net $\otimes_{n} \mathcal{A}_{n}$ together with the corresponding tensor product representation of $\mathrm{Möb}$ is not diffeomorphism covariant.

Proof. We denote by $V_{n}$ the representation of $\operatorname{Diff}^{+}\left(S^{1}\right)$ corresponding to $\mathcal{A}_{n}, n \in \mathbb{N}$, and by $c_{n}$ its central charge. For every positive integer $k$ the net $\otimes_{n} \mathcal{A}_{n}$ is isomorphic to

$$
\left(\mathcal{A}_{1} \otimes \ldots \otimes \mathcal{A}_{k}\right) \otimes \mathcal{B}_{k}
$$

where

$$
\mathcal{B}_{k}:=\bigotimes_{n>k} \mathcal{A}_{k}
$$

Let us assume that $\otimes_{n} \mathcal{A}_{n}$ is diffeomorphism covariant and let $V$ be the corresponding representation of $\operatorname{Diff}^{+}\left(S^{1}\right)$. By Prop. 6.1 there is a positive energy representation $V_{\mathcal{B}_{k}}$ of $\operatorname{Diff}^{+}\left(S^{1}\right)$ making $\mathcal{B}_{k}$ diffeomorphism covariant and such that

$$
V=V_{1} \otimes \ldots \otimes V_{k} \otimes V_{\mathcal{B}_{k}}
$$

Hence the central charge $c$ of $V$ satisfies

$$
c=c_{1}+\ldots+c_{k}+c\left(\mathcal{B}_{\mathrm{K}}\right) \geq \frac{k}{2}
$$

where $c\left(\mathcal{B}_{\mathrm{K}}\right)$ is the central charge of $V_{\mathcal{B}_{\mathrm{K}}}$, since the minimal possible value for a central charge is $1 / 2$. However, by the arbitrariness of $k$ we have a contradiction and the conclusion follows.

The examples of non diffeomorphism covariant nets considered in [19] are not strongly additive. However, they satisfy the trace class condition, namely $e^{-\beta L_{0}}$ is a trace class operator for every $\beta>0$ and hence they have the split property by [5. Theorem 3.2]. Conversely one can use Theorem 6.2 to give many examples of non diffeomorphism covariant strongly additive Möbius covariant nets on $S^{1}$. In these examples the operator $e^{-\beta L_{0}}$ is not compact for every value of $\beta$ since the eigenvalue 2 of $L_{0}$ always appears with infinite multiplicity. In fact, e.g. if the sequence $c_{k}$ of the 
central charges contains a constant subsequence the infinite tensor product net $\otimes_{n} \mathcal{A}_{n}$ does not satisfy the split property as a consequence of [7, Theorem 9.2].

Acknowledgements. We would like to thank Roberto Longo for suggesting the problem on the uniqueness of the diffeomorphism symmetry and for useful discussions. Some of the results contained in this paper were announced by the second named author (M. W.) at the conference on "Operator Algebras and Mathematical Physics", held in Sinaia (Romania) in July, 2003. He would also like to thank the organizers for the invitation.

\section{References}

[1] Buchholz D., Schulz-Mirbach H.: Haag duality in conformal quantum field theory. Rev. Math. Phys. 2 (1990), 105-125.

[2] Carpi S.: Absence of subsystems for the Haag-Kastler net generated by the energymomentum tensor in two-dimensional conformal field theory. Lett. Math. Phys. 45 (1998), 259-267.

[3] Carpi, S.: Quantum Noether's theorem and conformal field theory: a study of some models. Rev. Math. Phys. 11 (1999), 519-532.

[4] Carpi S.: On the representation theory of Virasoro nets. Commun. Math. Phys. 244 (2004), 261-284.

[5] D’Antoni C., Longo R., Radulescu F.: Conformal nets, maximal temperature and and models from free probability. J. Operator Theory 45 (2001), 195-208.

[6] Di Francesco Ph., Mathieu P., Sénéchal D.: Conformal field theory. SpringerVerlag, Berlin-Heidelberg-New York, 1996.

[7] Doplicher S., Longo R.: Standard and split inclusions of von Neumann algebras. Invent. Math. 75 (1984), 493-536.

[8] Fredenhagen K., Jörß M.: Conformal Haag-Kastler nets, pointlike localized fields and the existence of operator product expansions. Commun. Math. Phys. 176 (1996), 541-554.

[9] Gabbiani F., Fröhlich J.: Operator algebras and conformal field theory. Commun. Math. Phys. 155 (1993), 569-640.

[10] Goodman R. and Wallach N. R.: Projective unitary positive-energy representations of Diff $\left(S^{1}\right)$. J. Funct. Anal. 63, (1985) 299-321.

[11] Guido D., Longo R.: The conformal spin and statistics theorem. Commun. Math. Phys. 181 (1996), 11-35. 
[12] Guido D., Longo R., Wiesbrock H.-W.: Extensions of conformal nets and superselection structures. Commun. Math. Phys. 192 (1998), 217-244.

[13] Haag R.: Local quantum physics. 2nd ed. Springer-Verlag, Berlin-Heidelberg-New York, 1996.

[14] Kac V. G., Raina A. K.: Bombay lectures on highest weight representations of infinite dimensional Lie algebras. World Scientific, Singapore, 1987.

[15] Katznelson Y.: An introduction to harmonic analysis. Dover Publications, New York, 1976.

[16] Kawahigashi Y., Longo R.: Classification local conformal nets. Case $c<1$. math.OA/0211141, to appear in Ann. Math.

[17] Kawahigashi Y., Longo R.: Classification of two-dimensional local conformal nets with $c<1$ and 2-cohomology vanishing for tensor categories. Commun. Math. Phys. 244 (2004), 63-97.

[18] Kawahigashi Y., Longo R., Müger M.: Multi-interval subfactors and modularity of representations in conformal field theory. Commun. Math. Phys. 219 (2001), 631-669.

[19] Köster S.: Absence of stress energy tensor in CFT 2 models. math-ph/0303053.

[20] Köster S.: Local nature of coset models. Rev. Math. Phys. 16 (2004), 353-382.

[21] Loke T.: Operator algebras and conformal field theory of the discrete series representation of $\operatorname{Diff}^{+}\left(S^{1}\right)$. PhD Thesis, University of Cambridge, 1994.

[22] Longo R., Xu F.: Topological sectors and a dichotomy in conformal field theory. To appear in Commun. Math. Phys.

[23] Milnor J.: Remarks on infinite-dimensional Lie groups. In B.S. De Witt and R. Stora Eds.: Relativity, groups and topology II. Les Houches, Session XL, 1983, Elsevier, Amsterdam, New York, 1984, pp. 1007-1057.

[24] Nelson E.: Time-ordered operator product of sharp-time quadratic forms. J. Funct. Anal. 11 (1972), 211-219.

[25] Reed M., Simon B.: Methods of modern mathematical physics I: Functional analysis. Revised enlarged edition. Academic Press, San Diego-London, 1980.

[26] Reed M., Simon B.: Methods of modern mathematical physics II: Fourier analysis, self-adjointness. Academic Press, San Diego-London, 1975.

[27] Toledano Laredo V.: Fusion of positive energy representations of $\operatorname{LSpin}_{2 n}$. PhD Thesis, University of Cambridge, 1997. 
[28] Toledano Laredo V.: Integrating unitary representations of infinite-dimensional Lie groups. J. Funct. Anal. 161 (1999), 478-508.

[29] von Neumann J.: On infinite direct products. Compositio Math. 6 (1938), 1-77.

[30] Wassermann A.: Operator algebras and conformal field theory III: Fusion of positive energy representations of SU(N) using bounded operators. Invent. Math. 133 (1998) 467-538.

[31] Xu F.: Strong additivity and conformal nets. math.QA/0303266. 